\title{
Pauli reductions of supergravities in six and five dimensions
}

\author{
Arash Azizi ${ }^{1, *}$ and C. N. Pope ${ }^{1,2, \dagger}$ \\ ${ }^{1}$ George P. and Cynthia Woods Mitchell Institute for Fundamental Physics and Astronomy, \\ Texas A\&M University, College Station, Texas 77843, USA \\ ${ }^{2}$ DAMTP, Center for Mathematical Sciences, Cambridge University, Wilberforce Road, \\ Cambridge CB3 OWA, United Kingdom
}

(Received 25 June 2018; published 13 August 2018)

\begin{abstract}
The dimensional reduction of a generic theory on a curved internal space such as a sphere does not admit a consistent truncation to a finite set of fields that includes the Yang-Mills gauge bosons of the isometry group. In rare cases, e.g., the $S^{7}$ reduction of 11-dimensional supergravity, such a consistent "Pauli reduction" does exist. In this paper, we study this existence question in two examples of $S^{2}$ reductions of supergravities. We do this by making use of a relation between certain $S^{2}$ reductions and group manifold $S^{3}=S U(2)$ reductions of a theory in one dimension higher. By this means, we establish the nonexistence of a consistent $S^{2}$ Pauli reduction of five-dimensional minimal supergravity. We also show that a previously discovered consistent Pauli reduction of six-dimensional Salam-Sezgin supergravity can be elegantly understood via a group-manifold reduction from seven dimensions.
\end{abstract}

DOI: 10.1103/PhysRevD.98.046010

\section{INTRODUCTION}

The idea of using a dimensional reduction on a curved space such as a sphere in order to obtain a lowerdimensional theory with non-Abelian gauge symmetries has a long history, which seems to have originated with an unpublished communication by Pauli in 1952 [1,2]. His idea was that by dimensionally reducing six-dimensional Einstein gravity on the 2 sphere, one might obtain a fourdimensional theory describing gravity coupled to gauge fields that we would now call $S O(3)$ Yang-Mills. Pauli realized, however, that there were difficulties with this idea, which we can recognize as being inconsistencies in the higher-dimensional components of the Einstein equations. This is in fact a classic example of an inconsistent truncation in Kaluza-Klein.

One can always, of course, make a consistent KaluzaKlein "reduction" in which one simply expands all the higher-dimensional fields in terms of the complete set of harmonics (spherical harmonics, in the case of $S^{2}$ ) and retains the entire infinite towers of lower-dimensional massless and massive fields. The consistency issue arises if one wants to set all except a subset of the

\footnotetext{
sazizi@physics.tamu.edu

pope@physics.tamu.edu
}

Published by the American Physical Society under the terms of the Creative Commons Attribution 4.0 International license. Further distribution of this work must maintain attribution to the author(s) and the published article's title, journal citation, and DOI. Funded by SCOAP ${ }^{3}$. lower-dimensional fields to zero. For this to be a consistent truncation, it is necessary that the full set of equations of motion for the infinite towers of fields should be compatible with setting to zero the fields one wishes to truncate. The danger is that, because of the nonlinear nature of the theory, the fields one is retaining might act as sources for the fields one wishes to set to zero. This is exactly what happens in the case Pauli considered; the Yang-Mills fields one wishes to retain actually act as sources for a set of massive spin-2 fields that one wishes to set to zero. From the higher-dimensional point of view, this reflects itself as an inconsistency between the truncation Ansatz and the higher-dimensional components of the Einstein equations.

The inconsistency of the truncation to a finite number of lower-dimensional fields that include gravity and the YangMills fields of the isometry group of the compactifying manifold is not restricted to the case where one starts from pure higher-dimensional gravity. It is in fact what happens generically when compactifying any higher-dimensional theory that includes gravity. There are, however, some notable exceptions, of which the most celebrated is the reduction of 11-dimensional supergravity on the 7 sphere. In this case, the inconsistencies that would arise if 11dimensional pure gravity alone were reduced on $S^{7}$ are removed, because of further contributions in the higherdimensional equations of motion coming from the 3 -form potential. The consistency of this remarkable reduction was demonstrated in [3]. The exceptional situations where a consistent reduction on a curved internal manifold $M$, retaining a finite number of fields including gravity and the Yang-Mills gauge fields of the isometry group of $M$, have 
been called consistent Pauli reductions [4]. Other examples include the consistent Pauli reduction of 11-dimensional supergravity on $S^{4}$ [5-7], the consistent Pauli reduction of type IIB supergravity on $S^{5}$ [8], and the consistent Pauli reduction of the $D$-dimensional bosonic string on any group manifold $G$ [9]. In this latter case, we emphasize that the Pauli reduction on the group manifold $G$ yields the Yang-Mills gauge bosons of the full isometry group $G \times G$.

Establishing the consistency of the Pauli reductions in all the above examples is highly nontrivial. There is no simple group-theoretic argument for why the truncation to the finite subset of lower-dimensional fields should be consistent. This is in contrast to the very well understood class of "groupmanifold reductions" on $G$, in which the infinite towers of lower-dimensional fields are truncated to just those that are singlets under the right action $G_{R}$ (or, equivalently instead, the left action $G_{L}$ ) of $G$. These reductions were first described by DeWitt [10] and have been named DeWitt reductions in [4]. The consistency of the truncation follows trivially from the fact that the retained fields, which are singlets under $G_{R}$, clearly cannot act as sources for the nonsinglet fields that have all been set to zero.

In this paper, we shall investigate the consistent Pauli reduction on the 2 sphere of certain five-dimensional and six-dimensional supergravity theories. A common theme in both of these examples will be our employment of the observation made in [4] that in certain cases one can construct an $S^{2}$ Pauli reduction of a particular $D$-dimensional theory if that theory can itself be obtained from a circle reduction of a $(D+1)$-dimensional theory. If one starts by constructing the DeWitt $S U(2)$ reduction of the $(D+1)$ dimensional theory and makes a circle reduction on the Hopf fibers of $S U(2)$ viewed as a $U(1)$ bundle over $S^{2}$, then one obtains a (necessarily consistent) $S^{2}$ Pauli reduction of the $D$-dimensional theory. In the two examples that we shall be considering in this paper, a further complication arises because the $D$-dimensional theories of interest to us are in fact themselves consistent truncations of the circle reductions of the parent $(D+1)$-dimensional theories. That is to say, the truncation of fields can be performed in a $D$ dimensionally covariant way, but not in a $(D+1)$ dimensionally covariant way. This imposes a nontrivial further condition if one wishes to construct the $S^{2}$ Pauli reduction in the way we have described, since the additional field truncation in $D$ dimensions may not be compatible with the Hopf bundle structure. In fact, in one of our two examples, the Hopf construction will fail for this reason, while in the other example it succeeds.

Our first example is motivated by the well-known observation that the minimal five-dimensional supergravity, whose bosonic Lagrangian is

$\mathcal{L}_{5}=R * \mathbb{1}-\frac{1}{2} * F_{(2)} \wedge F_{(2)}-\frac{1}{3 \sqrt{3}} F_{(2)} \wedge F_{(2)} \wedge A_{(1)}$, where $F_{(2)}=d A_{(1)}$, is very closely parallel to 11-dimensional supergravity, whose bosonic Lagrangian is

$\mathcal{L}_{11}=R * \mathbb{1}-\frac{1}{2} * F_{(4)} \wedge F_{(4)}-\frac{1}{6} F_{(4)} \wedge F_{(4)} \wedge A_{(3)}$,

where $F_{(4)}=d A_{(3)}$. One is then tempted to conjecture that what works for $S^{4}$ or $S^{7}$ reductions of 11-dimensional supergravity would work also for $S^{2}$ or $S^{3}$ reductions of the minimal five-dimensional supergravity. In particular, one might expect that a consistent $S^{2}$ Pauli reduction of the minimal five-dimensional theory should be possible.

We shall study this question by making use of the construction developed in [4], which we described above. Thus, we shall take as our starting point the minimal supergravity of six dimensions, whose bosonic sector comprises gravity and a self-dual 3 form. The circle reduction of this theory gives minimal five-dimensional supergravity coupled to a single vector multiplet. Thus, we are able to construct a consistent $S^{2}$ Pauli reduction of this five-dimensional theory. Now the minimal fivedimensional supergravity itself arises as a consistent truncation of this theory, in which the vector multiplet is set to zero. However, as we indicated above, it is not guaranteed that imposing this truncation of the fivedimensional theory is itself compatible with the previously established consistent $S^{2}$ Pauli reduction, and in fact, we are able to show that the two conditions are inconsistent in this example. Although this does not constitute a complete proof that there exists no possible $S^{2}$ Pauli reduction of the five-dimensional minimal supergravity, the fact that this natural way to try to construct such a reduction fails is strongly suggestive. In fact other, direct, attempts to construct a consistent $S^{2}$ Pauli reduction of the five-dimensional minimal supergravity have also been unsuccessful.

Our second example is provided by the six-dimensional gauged supergravity of Salam and Sezgin [11]. This has the intriguing feature that it admits a supersymmetric $S^{2} \times$ (Minkowski) ${ }_{4}$ vacuum. Furthermore, it was shown in [12] that there is in fact a consistent $S^{2}$ Pauli reduction of the Salam-Sezgin model, which yields a four-dimensional supergravity with $S U(2)$ Yang-Mills fields originating from the isometry group of the 2 sphere, and whose Minkowski vacuum corresponds to the six-dimensional $S^{2} \times(\text { Minkowski })_{4}$ vacuum found in [11]. Although it is a much simpler example than the $S^{7}$ or $S^{4}$ reductions of $D=$ 11 supergravity, the underlying reasons for the consistency of this $S^{2}$ Pauli reduction are at present equally mysterious.

In this paper, we examine whether it is possible to reconstruct the $S^{2}$ Pauli reduction by exploiting the fact that the Salam-Sezgin supergravity can itself be obtained from a circle reduction of a seven-dimensional supergravity. Such 
an embedding of the Salam-Sezgin theory was obtained in [13], with the seven-dimensional theory being a noncompact gauged $S O(2,2)$ supergravity. A further field truncation is then required in six dimensions. However, for our present purposes, this embedding is not useful, because crucially the Kaluza-Klein vector of the circle reduction from seven to six dimensions is in fact set to zero. This means the seven-dimensional lift of the $S^{2}$ Pauli reduction would merely give an $S^{1} \times S^{2}$ reduction, with the consistency of the $S^{2}$ reduction remaining unexplained. Upon further investigation, we find that, at least at the bosonic level, there is in fact a different way to embed the Salam-Sezgin theory into the seven-dimensional $\operatorname{SO}(2,2)$ gauged supergravity, in which the Kaluza-Klein vector plays an active role. In this new embedding, it supplies the necessary twist of the $S^{1}$ fibers so that the lift of the $S^{2}$ Pauli reduction now becomes an $S U(2)$ DeWitt reduction from seven dimensions. Furthermore, we find that in this case the necessary additional truncation of fields in six dimensions is compatible with the structure of the Hopf fibration, and so we are able to reconstruct the $S^{2}$ Pauli reduction that was obtained in [12] as a DeWitt reduction from seven dimensions, and thus now with an understanding of why it works.

\section{II. $S^{1}$ REDUCTION OF MINIMAL $D=6$ SUPERGRAVITY}

Our starting point is the bosonic sector of minimal six-dimensional supergravity, for which the equations of motion are

$\hat{R}_{M N}=\frac{1}{8} \hat{H}_{M}^{P Q} \hat{H}_{N P Q}, \quad d \hat{H}_{(3)}=0, \quad \hat{*} \hat{H}_{(3)}=\hat{H}_{(3)}$.

Note that since $\hat{H}_{(3)}=d \hat{B}_{(2)}$ is self dual, one cannot write a six dimensionally covariant Lagrangian for the theory. We then perform a Kaluza-Klein $S^{1}$ reduction, using the standard Ansatz

$$
\begin{aligned}
d \hat{s}_{6}^{2} & =e^{2 \bar{\alpha} \phi} d \bar{s}_{5}^{2}+g^{-2} e^{2 \bar{\beta} \phi}(d \tau+g A)^{2}, \\
\hat{B}_{(2)} & =B_{(2)}+g^{-1} B_{(1)} \wedge d \tau,
\end{aligned}
$$

where we choose $\bar{\alpha}^{2}=1 / 24$ and $\bar{\beta}=-3 \bar{\alpha}$ in order to get the five-dimensional theory in the Einstein frame and with the canonical normalization for the dilaton field $\phi$. The constant $g$ that we have introduced here has the dimensions of (length $)^{-1}$ and serves the purpose of scaling the dimensionless coordinate $\tau$ that parametrizes the circle of the sixth dimension, so as to give a coordinate $z=g^{-1} \tau$ with the dimensions of length. Note that we are using hats to denote fields in the original six-dimensional theory. We place bars on five-dimensional quantities in cases where it is appropriate to distinguish them from six-dimensional quantities.

The Ansatz for $\hat{B}_{(2)}$ implies that we shall have

$$
\hat{H}_{(3)}=H_{(3)}+g^{-1} H_{(2)} \wedge(d \tau+g A),
$$

where

$$
H_{(3)}=d B_{(2)}-d B_{(1)} \wedge A, \quad H_{(2)}=d B_{(1)} .
$$

Since the 6 dual of $\hat{H}_{(3)}$ is given by

$$
\hat{*} \hat{H}_{(3)}=-g^{-1} e^{-4 \bar{\alpha} \phi_{\bar{*}} H_{(3)}} \wedge(d \tau+g A)+e^{4 \bar{\alpha} \phi_{\bar{*}} H_{(2)}},
$$

the six-dimensional self-duality condition $\hat{*} \hat{H}_{(3)}=\hat{H}_{(3)}$ implies the five-dimensional condition

$$
H_{(3)}=e^{4 \bar{\alpha} \phi_{\bar{*}} H_{(2)},}
$$

so the reduction (2.3) becomes

$$
\hat{H}_{(3)}=e^{4 \bar{\alpha} \phi} \bar{*} H_{(2)}+g^{-1} H_{(2)} \wedge(d \tau+g A),
$$

for the case of the self-dual $\hat{H}_{(3)}$.

The five-dimensional equations of motion resulting from substituting the reduction Ansätze into the six-dimensional equations (2.1) can be derived from the Lagrangian

$$
\begin{aligned}
\mathcal{L}_{5}= & \bar{R} \bar{*} 1-\frac{1}{2} \bar{*} d \phi \wedge d \phi-\frac{1}{2} e^{-8 \bar{\alpha} \phi \bar{*} F_{(2)} \wedge F_{(2)}} \\
& -\frac{1}{2} e^{4 \bar{\alpha} \phi_{\bar{*}} H_{(2)}} \wedge H_{(2)}-\frac{1}{2} H_{(2)} \wedge H_{(2)} \wedge A_{(1)} .
\end{aligned}
$$

This Lagrangian describes the bosonic sector of fivedimensional minimal supergravity coupled to one vector multiplet. The truncation to pure minimal supergravity is then achieved by setting

$$
\phi=0, \quad B_{(1)}=\sqrt{2} A_{(1)},
$$

which can easily be seen to be consistent with the equations of motion. If we define $\tilde{A}_{(1)}=\sqrt{3} A_{(1)}$ so that the remaining gauge field has a canonical normalization, the Lagrangian (2.8) reduces to that for the bosonic sector of pure minimal supergravity,

$$
\mathcal{L}_{5}=\bar{R} \bar{*} 1-\frac{1}{2} \bar{*} \tilde{F}_{(2)} \wedge \tilde{F}_{(2)}-\frac{1}{3 \sqrt{3}} \tilde{F}_{(2)} \wedge \tilde{F}_{(2)} \wedge \tilde{A}_{(1)} .
$$




\section{III. $S U(2)$ DEWITT REDUCTION FROM $D=6$ TO $D=3$}

\section{A. Description as an $S U(2)$ group manifold reduction}

The $S U(2)$ group manifold DeWitt reduction of minimal six-dimensional supergravity was constructed in [14]. The reduction Ansatz for the metric and the self-dual 3 form are given by

$$
\begin{gathered}
d \hat{s}_{6}^{2}=e^{2 \alpha \varphi} d s_{3}^{2}+g^{-2} e^{2 \beta \varphi} \tilde{T}_{i j} \nu^{i} \nu^{j} \\
\hat{H}_{(3)}=m g^{-3} \Omega_{(3)}+m e^{4 \alpha \varphi} \epsilon_{(3)}+\frac{1}{2} g^{-2} \varepsilon_{i j k} B^{i} \wedge \nu^{j} \wedge \nu^{k} \\
-g^{-1} e^{\frac{4 \alpha \varphi}{3}} \tilde{T}_{i j} * B^{i} \wedge \nu^{j},
\end{gathered}
$$

where the constants $\alpha$ and $\beta$ are taken to be given by $\alpha^{2}=$ $3 / 8$ and $\beta=-\alpha / 3$ in order to obtain the three-dimensional theory in Einstein frame with the canonical normalization for the dilaton $\varphi$. The unimodular matrix $\tilde{T}_{i j}$ parametrizes the remaining scalar fields of the three-dimensional theory, and the 1 forms

$$
\nu^{i}=\sigma^{i}-g A^{i}
$$

are written in terms of the left-invariant 1 forms $\sigma^{i}$ of $S U(2)$ and the $S U(2)$ Yang-Mills potentials $A^{i}$, and

$$
\Omega_{(3)} \equiv \nu^{1} \wedge \nu^{2} \wedge \nu^{3},
$$

$\epsilon_{(3)}$ is the volume form of the three-dimensional spacetime, and $B^{i}$ denotes an $S U(2)$ triplet of 1-form fields.

The $\sigma^{i}$, which can be expressed in terms of Euler angles $(\psi, \theta, \tau)$ as

$$
\begin{aligned}
& \sigma_{1}=\cos \psi d \theta+\sin \psi \sin \theta d \tau, \\
& \sigma_{2}=-\sin \psi d \theta+\cos \psi \sin \theta d \tau, \\
& \sigma_{3}=d \psi+\cos \theta d \tau,
\end{aligned}
$$

obey the relations

$$
d \sigma_{i}=-\frac{1}{2} \varepsilon_{i j k} \sigma_{j} \wedge \sigma_{k}
$$

We also have

$$
D \nu^{i}=-\frac{1}{2} \varepsilon_{i j k} \nu^{j} \wedge \nu^{k}-g F^{i},
$$

where

$$
F^{i}=d A^{i}+\frac{1}{2} g \varepsilon_{i j k} A^{j} \wedge A^{k}, \quad D \nu^{i} \equiv d \nu^{i}+g \varepsilon_{i j k} A^{j} \wedge \nu^{k} .
$$

Note that the equation $d \hat{H}_{(3)}=0$ implies that

$$
D B^{i}-m F^{i}+g e^{\frac{4}{3} \alpha \varphi} \tilde{T}_{i j} * B^{j}=0,
$$

where $D B^{i}=d B^{i}+g \varepsilon_{i j k} A^{j} \wedge B^{k}$.

The equations of motion for the three-dimensional theory, obtained by substituting (3.1) and (3.2) into (2.1), can be derived from a Lagrangian whose precise form can be found in [14].

\section{B. $S U(2)$ as a Hopf fibration}

Following [4], we may now rewrite the $S U(2)$ DeWitt reduction of Sec. III A in a form where $S U(2)$ is viewed as a $U(1)$ Hopf fibration over $S^{2}$. Thus, we describe the unit $S^{2}$ as the surface $\mu_{1}^{2}+\mu_{2}^{2}+\mu_{3}^{2}=1$ in $\mathbb{R}^{3}$, where the three Cartesian coordinates $\mu_{i}$ are parametrized in terms of the $\theta$ and $\psi$ Euler angles introduced in (3.5) by

$\mu_{1}=\sin \psi \sin \theta, \quad \mu_{2}=\cos \psi \sin \theta, \quad \mu_{3}=\cos \theta$.

The 1-forms $\nu^{i}$ defined in (3.3) can then be written as [4]

$$
\begin{aligned}
\nu^{i} & =\sigma^{i}-g A^{i}=-\varepsilon_{i j k} \mu^{j} D \mu^{k}+\mu^{i} \sigma, \\
\sigma & \equiv d \tau+\cos \theta d \psi-g \mu^{i} A^{i},
\end{aligned}
$$

where the covariant derivative is defined as $D \mu^{i} \equiv$ $d \mu^{i}+g \varepsilon_{i j k} A^{j} \mu^{k}$.

The metric reduction Ansatz (3.1) can now be seen to be given by [4]

$$
\begin{aligned}
d \hat{s}_{6}^{2}= & e^{2 \alpha \varphi} d s_{3}^{2}+g^{-2} e^{2 \beta \varphi} \tilde{\Delta}^{-1} \tilde{T}_{i j}^{-1} D \mu^{i} D \mu^{j} \\
& +g^{-2} e^{2 \beta \varphi} \tilde{\Delta}(d \tau+g A)^{2}
\end{aligned}
$$

where

$A=g^{-1} \cos \theta d \psi-\mu^{i} A^{i}-g^{-1} \tilde{\Delta}^{-1} \tilde{T}_{i j} \varepsilon_{i k \ell} \mu^{j} \mu^{k} D \mu^{\ell}$,

$\tilde{\Delta}=\tilde{T}_{i j} \mu^{i} \mu^{j}$.

After some algebra, we find we can write

$$
\begin{aligned}
\nu^{i} & =\mu^{i}(d \tau+g A)-\tilde{\Delta}^{-1} \tilde{T}_{j k} \varepsilon_{i j \ell} \mu^{k} D \mu^{\ell}, \\
\frac{1}{2} \varepsilon_{i j k} \nu^{j} \wedge \nu^{k} & =(d \tau+g A) \wedge D \mu^{i}+\tilde{\Delta}^{-1} \tilde{T}_{i j} \mu^{j} \omega_{(2)},
\end{aligned}
$$

$$
\Omega_{(3)}=\frac{1}{6} \varepsilon_{i j k} \nu^{i} \wedge \nu^{j} \wedge \nu^{k}=(d \tau+g A) \wedge \omega_{(2)},
$$

where 


$$
\omega_{(2)}=\frac{1}{2} \varepsilon_{i j k} \mu^{i} D \mu^{j} \wedge D \mu^{k} .
$$

It then follows that the reduction Ansatz (3.2) for the selfdual 3 form is given by

$$
\begin{aligned}
\hat{H}_{(3)}= & (d \tau+g A) \\
& \wedge\left[m g^{-3} \omega_{(2)}-g^{-2} B^{i} \wedge D \mu^{i}-g^{-1} e^{\frac{4}{3} \alpha \varphi} \tilde{T}_{i j} \mu^{i} * B^{j}\right] \\
& +m e^{4 \alpha \varphi} \epsilon_{(3)}+g^{-2} \tilde{\Delta}^{-1} \tilde{T}_{i j} \mu^{i} B^{j} \wedge \omega_{(2)} \\
& +g^{-1} e^{\frac{4}{3} \alpha \varphi} \tilde{\Delta}^{-1} \varepsilon_{j k m} \mu^{\ell} \tilde{T}_{i j} \tilde{T}_{k \ell} * B^{i} \wedge D \mu^{m}
\end{aligned}
$$

With these preliminaries, we are now ready to reinterpret the DeWitt $S U(2)$ group manifold reduction of the minimal six-dimensional supergravity as a Pauli $S^{2}$ reduction from five dimensions. To do this, we compare the expressions (2.2) and (2.7) for the $S^{1}$ reduction with the corresponding expressions (3.12) and (3.16) for the $S U(2)$ reduction expressed in the notation of the Hopf fibration. Thus, from the comparison of the metrics, we find

$$
\begin{gathered}
d \bar{s}_{5}^{2}=e^{2 \alpha \varphi-2 \bar{\alpha} \phi} d s_{3}^{2}+g^{-2} e^{-\frac{2}{3} \alpha \varphi-2 \bar{\alpha} \phi} \tilde{\Delta}^{-1} \tilde{T}_{i j}^{-1} D \mu^{i} D \mu^{j}, \\
e^{-6 \bar{\alpha} \phi}=e^{-\frac{2}{3} \alpha \varphi} \tilde{\Delta}
\end{gathered}
$$

and from the comparison of the reduction Ansätze for the six-dimensional self-dual 3 -form $\hat{H}_{(3)}$, we find

$$
\begin{aligned}
H_{(2)}= & m g^{-2} \omega_{(2)}-g^{-1} B^{i} \wedge D \mu^{i}-e^{\frac{4}{3} \alpha \varphi} \tilde{T}_{i j} \mu^{i} * B^{j}, \\
e^{4 \bar{\alpha} \phi} \bar{*}_{\bar{*} H_{(2)}=} & m e^{4 \alpha \varphi} \epsilon_{(3)}+g^{-2} \tilde{\Delta}^{-1} \tilde{T}_{i j} \mu^{i} B^{j} \wedge \omega_{(2)} \\
& +g^{-1} e^{\frac{4}{3} \alpha \varphi} \tilde{\Delta}^{-1} \varepsilon_{j k m} \mu^{\ell} \tilde{T}_{i j} \tilde{T}_{k \ell^{*}} * B^{i} \wedge D \mu^{m} .
\end{aligned}
$$

Following [4], we now define the three-dimensional scalar fields

$$
T_{i j}=Y^{\frac{1}{3}} \tilde{T}_{i j}, \quad Y=e^{4 \alpha \varphi},
$$

in terms of which the Pauli reduction Ansätze for the fivedimensional metric $d \bar{s}_{5}^{2}$ and fields $\phi, A$, and $H_{(2)}=d B_{(1)}$ become

$$
\begin{aligned}
d \bar{s}_{5}^{2} & =Y^{\frac{1}{3}} \Delta^{\frac{1}{3}} d s_{3}^{2}+g^{-2} Y^{\frac{1}{3}} \Delta^{-\frac{2}{3}} T_{i j}^{-1} D \mu^{i} D \mu^{j}, \\
e^{6 \bar{\alpha} \phi} & =Y^{\frac{1}{2}} \Delta^{-1}, \\
A & =g^{-1} \cos \theta d \psi-\mu^{i} A^{i}-g^{-1} \Delta^{-1} T_{i j} \varepsilon_{i k \ell} \mu^{j} \mu^{k} D \mu^{\ell}, \\
H_{(2)} & =m g^{-2} \omega_{(2)}-g^{-1} B^{i} \wedge D \mu^{i}-T_{i j} \mu^{i} * B^{j},
\end{aligned}
$$

where $\Delta=T_{i j} \mu^{i} \mu^{j}=Y^{\frac{1}{3}} \tilde{\Delta}$.

Making use of the Eq. (3.9), we can see that $H_{(2)}=$ $d B_{(1)}$ given in (3.21) can be written as

$$
H_{(2)}=m g^{-2} \omega_{(2)}-m g^{-1} \mu^{i} F^{i}+g^{-1} d\left(\mu^{i} B^{i}\right),
$$

and hence, $B_{(1)}$ can be written explicitly as

$$
B_{(1)}=m g^{-2} \cos \theta d \psi-m g^{-1} \mu^{i} A^{i}+g^{-1} \mu^{i} B^{i} .
$$

\section{PAULI REDUCTION OF 5D MINIMAL SUPERGRAVITY?}

In Sec. III B, we constructed the consistent $S^{2}$ Pauli reduction of the bosonic sector of the five-dimensional supergravity that is obtained by means of the $S^{1}$ KaluzaKlein reduction of minimal six-dimensional supergravity. As we showed in Sec. II, the five-dimensional theory can be truncated to give the bosonic sector of pure minimal fivedimensional supergravity by imposing the conditions (2.9) on the five-dimensional fields. In this section, we address the question of whether we can consistently impose this truncation on the three-dimensional fields in the Pauli reduction in Sec. III B, thereby obtaining a consistent $S^{2}$ Pauli reduction of five-dimensional minimal supergravity.

From the Pauli reduction Ansatz for $\phi$ given in (3.21), we see that setting $\phi=0$ requires imposing

$$
Y^{\frac{1}{2}}=\Delta=T_{i j} \mu^{i} \mu^{j} .
$$

Since $Y$ and $T_{i j}$ are three-dimensional fields, which cannot depend on the $S^{2}$ coordinates $\mu^{i}$, it follows that we must have

$$
T_{i j}=f \delta_{i j},
$$

where $f$ is a function only of the three-dimensional fields. Taking the determinant of $T_{i j}$, and noting from (3.20) that it must equal $Y$, we then conclude that $f^{2}=f^{3}$ and hence, $f=1$, so $T_{i j}=\delta_{i j}$. From the reduction Ansätze for $A$ and $B_{(1)}$ given in (3.21) and (3.23), we then conclude that making the truncation $B_{(1)}=\sqrt{2} A$ in (2.9) implies

$$
m=\sqrt{2} g, \quad B^{i}=0 .
$$

Finally, from (3.9), we see that $B^{i}=0$ implies that $F^{i}=0$, and so $A^{i}$ is pure gauge.

The conclusion from the above discussion is that one cannot truncate the consistent $S^{2}$ Pauli reduction of the full five-dimensional theory to give a consistent Pauli reduction of the five-dimensional minimal supergravity theory.

\section{SALAM-SEZGIN THEORY BY $\boldsymbol{S}^{1}$ REDUCTION FROM $D=7$}

First, we need to see how to obtain the $D=6$ SalamSezgin theory from an $S^{1}$ reduction of a $D=7$ theory. 
In [7], the details of the $S^{3}$ reduction from $D=10$, giving $\mathcal{N}=4$ supersymmetric gauged $S O(4)$ sugra in $D=7$ were given; it was obtained as a limit of the gauged $S O(5)$ supergravity that comes from $S^{4}$ reduction from $D=11$. The $S O(4) \rightarrow S O(2,2)$ replacement was then discussed in [13], where it was then shown how Salam-Sezgin could be obtained via an $S^{1}$ reduction of gauged $S O(2,2) \mathcal{N}=2$ supergravity by $S^{1}$ reduction followed by a consistent truncation. Prior to the final consistent truncation in $D=6$, the bosonic Lagrangian is given by Eq. (30) of [13]. We can straightforwardly make a truncation of all $S O(2,2)$ fields to those that are singlets under the $U(1) \times$ $U(1)$ maximal subgroup. The truncated seven-dimensional bosonic Lagrangian from which the reduction to six dimensions can be obtained is

$$
\begin{aligned}
\mathcal{L}_{7}= & \hat{R} \hat{*} \mathbb{1}-\frac{5}{16} \hat{\Phi}^{-2} \hat{d} \hat{\Phi} \wedge d \hat{\Phi}-\hat{\Phi}^{-1 / 2}\left(* \hat{F}_{(2)}^{12} \wedge \hat{F}_{(2)}^{12}\right. \\
& \left.+* \hat{F}_{(2)}^{34} \wedge \hat{F}_{(2)}^{34}\right)-\frac{1}{2} \hat{\Phi}^{-1} \hat{*} \hat{H}_{(3)} \wedge \hat{H}_{(3)} \\
& -4 g^{2} \hat{\Phi}^{\frac{1}{2}} \hat{\mathbb{1}}+\mathcal{L}_{7, \mathrm{CS}},
\end{aligned}
$$

where the $S O(2,2)$ gauge potentials $\hat{A}_{(1)}^{\alpha \beta}$ have been truncated to just the Abelian subsector $\hat{A}_{(1)}^{12}$ and $\hat{A}_{(1)}^{34}$, and $\hat{H}_{(3)}=d \hat{B}_{(2)}+\hat{F}_{(2)}^{12} \wedge \hat{A}_{(1)}^{34}+\hat{F}_{(2)}^{34} \wedge \hat{A}_{(1)}^{12}$. Performing a Kaluza-Klein circle reduction in the usual way, by means of the Ansätze ${ }^{1}$

$$
\begin{aligned}
d \hat{s}_{7}^{2} & =e^{2 \alpha \varphi} d \bar{s}_{6}^{2}+e^{-8 \alpha \varphi}\left(d z+\mathcal{A}_{(1)}\right)^{2}, \\
\hat{B}_{(2)} & =\bar{B}_{(2)}+\bar{B}_{(1)} \wedge d z, \quad \hat{A}_{(1)}^{\alpha \beta}=A_{(1)}^{\alpha \beta}, \quad \hat{\Phi}=\Phi,
\end{aligned}
$$

where $\quad \alpha=1 /(2 \sqrt{10}), \quad$ gives the six-dimensional Lagrangian $^{2}$

$$
\begin{aligned}
& \mathcal{L}_{6}=\bar{R} \bar{\star} \mathbb{1}-\frac{5}{16} \Phi^{-2} \bar{*} d \Phi \wedge d \Phi-\frac{1}{2} \bar{*} d \varphi \wedge d \varphi \\
& -\frac{1}{2} e^{-10 \alpha \varphi_{\bar{*}} \mathcal{F}_{(2)}} \wedge \mathcal{F}_{(2)}-\frac{1}{2} \Phi^{-1} e^{6 \alpha \varphi_{\bar{*}} \bar{H}_{(2)}} \wedge \bar{H}_{(2)} \\
& -\Phi^{-1 / 2} e^{-2 \alpha \varphi}\left(\bar{*} F_{(2)}^{12} \wedge F_{(2)}^{12}+\bar{*} F_{(2)}^{34} \wedge F_{(2)}^{34}\right)
\end{aligned}
$$

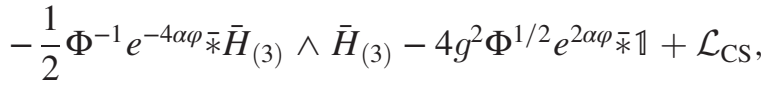

\footnotetext{
${ }^{1}$ We shall use a bar to denote six-dimensional quantities, such as the metric and $\bar{B}_{(2)}$, where we may need to distinguish them later from four-dimensional quantities, which will be unbarred. In cases where there is no possibility of confusion with fourdimensional quantities, we shall omit the bar, as, e.g., in the six-dimensional gauge fields $A_{(1)}^{12}$ and $A_{(1)}^{34}$.

${ }^{2}$ There were some typographical errors in [7], which we have corrected here, relating to the coefficient of the kinetic term for the scalar field $\Phi$, and also the form of the Chern-Simons term, whose variation is given by (5.5).
}

where

$$
\begin{aligned}
& \bar{H}_{(3)}=d \bar{B}_{(2)}-d \bar{B}_{(1)} \wedge \mathcal{A}_{(1)}+F_{(2)}^{12} \wedge A_{(1)}^{34}+F_{(2)}^{34} \wedge A_{(1)}^{12}, \\
& \bar{H}_{(2)}=d \bar{B}_{(1)}, \quad \mathcal{F}_{(2)}=d \mathcal{A}_{(1)},
\end{aligned}
$$

and $\bar{*}$ denotes the six-dimensional Hodge dual in the metric $d \bar{s}_{6}^{2}$. The term $\mathcal{L}_{\mathrm{CS}}$ is a Chern-Simons term, whose variation is given, up to a certain overall normalization constant $c$, by

$$
\begin{aligned}
\delta \mathcal{L}_{\mathrm{CS}}= & c\left(F_{(2)}^{\beta \gamma} \wedge F_{(2)}^{\gamma \delta} \wedge F_{(2)}^{\delta \alpha}-\frac{1}{4} F_{(2)}^{\gamma \delta} \wedge F_{(2)}^{\delta \gamma} \wedge F_{(2)}^{\beta \alpha}\right) \\
& \wedge \delta A_{(1)}^{\alpha \beta} .
\end{aligned}
$$

An important point for the consistency of the truncation that gives the Salam-Sezgin theory is that this ChernSimons contribution vanishes if one sets $A_{(1)}^{12}= \pm A_{(1)}^{34}$.

Note that we have, for reasons of presentational simplicity, omitted the axions that would come from the reduction of the seven-dimensional gauge fields $\hat{A}_{(1)}^{12}$ and $\hat{A}_{(1)}^{34}$. Setting them to zero would not in general be a consistent truncation, but it is consistent to do so in either of the two further truncations that we shall be considering below, namely either setting $A_{(1)}^{12}=-A_{(1)}^{34}$ with $\mathcal{A}_{(1)}=B_{(1)}=0$, or else setting $A_{(1)}^{12}=A_{(1)}^{34}=0$ with $\mathcal{A}_{(1)}=-B_{(1)}$.

It is convenient to reparametrize the scalars in terms of the two fields $\phi$ and $\psi$, where

$$
\Phi=e^{\frac{2}{5} \psi-\frac{4}{5} \phi}, \quad 20 \alpha \varphi=-2 \psi-\phi .
$$

In terms of these, the Lagrangian becomes

$$
\begin{aligned}
\mathcal{L}_{6}= & \bar{R} \bar{*} \mathbb{1}-\frac{1}{4} \bar{*} d \phi \wedge d \phi-\frac{1}{4} \bar{*} d \psi \wedge d \psi-\frac{1}{2} e^{\frac{1}{2} \phi+\psi} \bar{*} \mathcal{F}_{(2)} \\
& \wedge \mathcal{F}_{(2)}-\frac{1}{2} e^{\frac{1}{2} \phi-\psi} \bar{*} \bar{H}_{(2)} \wedge \bar{H}_{(2)} \\
& -e^{\frac{1}{2} \phi}\left(\bar{*} F_{(2)}^{12} \wedge F_{(2)}^{12}+\bar{*} F_{(2)}^{34} \wedge F_{(2)}^{34}\right)-\frac{1}{2} e^{\phi} \bar{*} \bar{H}_{(3)} \\
& \wedge \bar{H}_{(3)}-4 g^{2} e^{-\frac{1}{2} \phi} \bar{*} \mathbb{1}+\mathcal{L}_{\mathrm{CS}} .
\end{aligned}
$$

\section{A. Truncations to Salam-Sezgin theory}

It was shown in [13] that the Salam-Sezgin theory could be obtained by making a further, consistent, truncation of the six-dimensional supergravity whose relevant bosonic sector is described by (5.7). Namely, one now sets

$$
\begin{aligned}
& \mathcal{A}_{(1)}=0, \quad \bar{B}_{(1)}=0, \\
& A_{(1)}^{12}=-A_{(1)}^{34} \equiv \frac{1}{2} A_{(1)}, \quad \psi=0,
\end{aligned}
$$


leading to the Salam-Sezgin bosonic Lagrangian

$$
\begin{aligned}
& \mathcal{L}_{\mathrm{SS}}=\bar{R} \bar{*} \mathbb{1}-\frac{1}{4} \bar{*} d \phi \wedge d \phi-\frac{1}{2} e^{\frac{1}{2} \phi} \bar{*} F_{(2)} \wedge F_{(2)} \\
& -\frac{1}{2} e^{\phi} \bar{*} \bar{H}_{(3)} \wedge \bar{H}_{(3)}-4 g^{2} e^{-\frac{1}{2} \phi} \bar{*} \mathbb{1} .
\end{aligned}
$$

[Recall that, as already remarked, the setting to zero of the axions coming from the reduction of the $U(1) \times U(1)$ Yang-Mills potentials is consistent, once the truncation (5.8) is performed. Furthermore, the Chern-Simons contribution vanishes under this truncation.]

This construction, and its extension to include the fermionic sector also, was studied in detail in [13]. It gives a consistent embedding of the Salam-Sezgin theory in a seven-dimensional gauged supergravity, which in turn can be obtained as a consistent reduction of ten-dimensional supergravity. However, it does not provide us with a way to understand the occurrence of the consistent Pauli $S^{2}$ reduction [12] of the Salam-Sezgin theory itself. The understanding of a consistent Pauli $S^{2}$ reduction from $D$ dimensions by first considering a (trivially) consistent DeWitt $S U(2)$ group manifold reduction from $(D+1)$ dimensions depended upon the $S^{2}$ reduction becoming an $S U(2)$ reduction when lifted to the higher dimension. This depends upon the Kaluza-Klein vector of the $(D+1) \rightarrow D$ reduction providing the necessary nontrivial monopole background that twists the $S^{1}$ into a Hopf fibration over the $S^{2}$, becoming the $S U(2)$ group manifold. In the construction in [13], however, the Kaluza-Klein vector is actually set to zero, as in (5.8), and so the lift to $D=7$ of the consistent Pauli $S^{2}$ reduction of the Salam-Sezgin theory that was found in [12] will be an $S^{1} \times S^{2}$ reduction rather than an $S U(2)$ group-manifold reduction.

If we are to find an explanation of the consistency of the $S^{2}$ Pauli reduction of the Salam-Sezgin theory in terms of a Hopf reduction of an $S U(2)$ group manifold reduction from $D=7$, we must find a different embedding of the SalamSezgin theory into $D=7$, in which the Kaluza-Klein vector plays the role of supplying the necessary monopole twist. At least at the bosonic level, the existence of such an alternative reduction can be seen by looking again at the six-dimensional Lagrangian (5.7). Now, we set

$\mathcal{A}_{(1)}=-\bar{B}_{(1)} \equiv \frac{1}{\sqrt{2}} A_{(1)}, \quad A_{(1)}^{12}=A_{(1)}^{34}=0, \quad \psi=0$.

It is straightforward to check that this is indeed a consistent truncation and that it yields the same Salam-Sezgin bosonic Lagrangian (5.9) that we saw previously. [Again, the setting to zero of the axions coming from the reduction of the $U(1) \times U(1)$ gauge potentials is indeed consistent, under this truncation, and the Chern-Simons term again gives zero contribution.]

\section{DEWITT AND HOPF REDUCTION FROM $D=7$}

Having seen that we can indeed obtain the bosonic Salam-Sezgin theory from seven dimensions in a circle reduction where the Kaluza-Klein vector is active, we now turn to the question of whether we can use this to obtain the consistent Pauli $S^{2}$ reduction of Salam-Sezgin via a Hopf reduction of the seven-dimensional theory. The calculations here will be closely analogous to those that we carried out in Sec. III. Accordingly, we begin by considering the $S U$ (2) group manifold DeWitt reduction from $D=7$, with the standard metric Ansatz ${ }^{3}$

$$
d \hat{s}_{7}^{2}=e^{2 \alpha^{\prime} \varphi^{\prime}} d s_{4}^{2}+\frac{1}{4} g^{-2} e^{-\frac{4 \alpha^{\prime}}{3} \varphi^{\prime}} T_{i j} \nu^{i} \nu^{j}
$$

where $\nu^{i}=\sigma^{i}-g A^{i}$, with $\sigma^{i}$ being the left-invariant 1 forms of $S U(2)$, as described in Sec. III. A, and $\alpha^{2}=\frac{3}{20}$. The matrix of scalar fields $T_{i j}$ is unimodular.

Following the same strategy as we did in Sec. III, we now write this in terms of the Hopf fibration, which here will take the form

$$
\begin{aligned}
d \hat{s}_{7}^{2}= & e^{2 \alpha^{\prime} \varphi^{\prime}} d s_{4}^{2}+\frac{1}{4} g^{-2} e^{-\frac{4 \alpha^{\prime}}{3} \varphi^{\prime}} \Delta^{-1} T_{i j}^{-1} D \mu^{i} D \mu^{j} \\
& +e^{-\frac{4 \alpha^{\prime}}{3} \varphi^{\prime}} \Delta\left(d z+\mathcal{A}_{(1)}\right)^{2}
\end{aligned}
$$

where

$$
\begin{aligned}
\mathcal{A}_{(1)} & =\frac{1}{2} g^{-1} \cos \theta d \psi-\frac{1}{2} \mu^{i} A^{i}-\frac{1}{2} g^{-1} \Delta^{-1} T_{i j} \varepsilon_{i k \ell} \mu^{j} \mu^{k} D \mu^{\ell}, \\
\Delta & =T_{i j} \mu^{i} \mu^{j} .
\end{aligned}
$$

Comparing (6.2) with the $S^{1}$ reduction of the metric in (5.2), we see we must have

$$
e^{-8 \alpha \varphi}=e^{-\frac{4 \alpha^{\prime}}{3} \varphi^{\prime}} \Delta,
$$

together with

$d \bar{s}_{6}^{2}=e^{-2 \alpha \varphi+2 \alpha^{\prime} \varphi^{\prime}} d s_{4}^{2}+\frac{1}{4} g^{-2} e^{-2 \alpha \varphi-\frac{4 \alpha^{\prime}}{3} \varphi^{\prime}} \Delta^{-1} T_{i j}^{-1} D \mu^{i} D \mu^{j}$.

A straightforward calculation from (6.3) shows that

\footnotetext{
${ }^{3}$ Note that the vacuum solution $S^{3} \times(\text { Minkowski) })_{4}$ of the theory described by (5.1) has $d \hat{s}_{7}^{2}=d x^{\mu} d x_{\mu}+g^{-2} d \Omega_{3}^{2}$ together with $\hat{H}_{(3)}= \pm 2 g \tilde{\Omega}_{(3)}$ and $\hat{\Phi}=1$, where $d \Omega_{3}^{2}$ is the metric on the unit $S^{3}$ and $\tilde{\Omega}_{(3)}$ is its volume form. [Note that $\tilde{\Omega}_{(3)}=\frac{1}{8} \Omega_{(3)}$ in the vacuum, where $\Omega_{(3)}$ was defined earlier in Eq. (3.14).]
} 


$$
\begin{aligned}
\mathcal{F}_{(2)}= & -\frac{1}{2} g^{-1} U \Delta^{-2} \omega_{(2)}+\frac{1}{2} g^{-1} \Delta^{-2} \varepsilon_{i j k} D \mu^{i} \\
& \wedge D T_{j \ell} T_{\mathrm{km}} \mu^{\ell} \mu^{m}-\frac{1}{2} \Delta^{-1} T_{i j} \mu^{i} F^{j},
\end{aligned}
$$

where $U=2 T_{i k} T_{k j} \mu^{i} \mu^{j}-\Delta T_{i i}$.

The general DeWitt reduction of the 3 form in seven dimensions is given by

$$
\begin{aligned}
\hat{H}_{(3)}= & m g^{-3} \Omega_{(3)}+g^{-2} \frac{1}{2} \varepsilon_{i j k} B^{i} \wedge \nu^{j} \wedge \nu^{k} \\
& +g^{-1} C^{i} \wedge \nu^{i}+H_{(3)},
\end{aligned}
$$

where $\Omega_{(3)}$ was defined in (3.4), $B^{i}$, and $C^{i}$ are $S U(2)$ triplets of four-dimensional 1-form and 2-form fields, respectively, and $H_{(3)}$ is a four-dimensional 3 form. Using the relations

$$
\begin{aligned}
\nu^{i} & =2 g \mu^{i}\left(d z+\mathcal{A}_{(1)}\right)-\Delta^{-1} T_{j k} \varepsilon_{i j \ell} \mu^{k} D \mu^{\ell}, \\
\frac{1}{2} \varepsilon_{i j k} \nu^{j} \wedge \nu^{k} & =2 g\left(d z+\mathcal{A}_{(1)}\right) \wedge D \mu^{i}+\Delta^{-1} T_{i j} \mu^{j} \omega_{(2)}, \\
\Omega_{(3)} & =\frac{1}{6} \varepsilon_{i j k} \nu^{i} \wedge \nu^{j} \wedge \nu^{k}=2 g\left(d z+\mathcal{A}_{(1)}\right) \wedge \omega_{(2)},
\end{aligned}
$$

and comparing with the $S^{1}$ reduction for $\hat{B}_{2}$ in (5.2), and the six-dimensional definitions (5.4) [recall we are setting the seven-dimensional $U(1) \times U(1)$ gauge fields to zero in this truncation], we find that

$$
\begin{aligned}
\bar{H}_{(2)}= & 2 m g^{-2} \omega_{(2)}-2 g^{-1} B^{i} \wedge D \mu^{i}+2 \mu^{i} C^{i}, \\
\bar{H}_{(3)}= & g^{-2} \Delta^{-1} T_{i j} \mu^{j} B^{i} \wedge \omega_{(2)}-g^{-1} \Delta^{-1} T_{j k} \varepsilon_{i j \ell} \mu^{k} C^{i} \\
& \wedge D \mu^{\ell}+H_{(3)} .
\end{aligned}
$$

With these reduction Ansätze for the metric and the 3 form, we are thus able to give the complete DeWitt $S U(2)$ reduction of the seven-dimensional theory described by the Lagrangian (5.1) (with $\hat{A}_{(1)}^{12}=\hat{A}_{(1)}^{34}=0$ and hence, the Chern-Simons term making no contribution either). The reduction is expressed in the form of the Hopf fibration, allowing us to make contact with the $S^{1}$ reduction we discussed earlier. Specifically, we now wish to impose the further truncation of the six-dimensional fields given in (5.10), which takes us in six dimensions to the SalamSezgin theory.

As we saw in a similar discussion in Sec. III, it is not guaranteed that imposing this truncation will be compatible with retaining the desired fields in the DeWitt-Hopf reduction. The key question here is whether imposing the truncation $\mathcal{F}_{(2)}=-H_{(2)}$ is compatible with the expressions in (6.6) and (6.9) for these fields. Equating the two expressions, we find
$U=4 m g^{-1} \Delta^{2}, \quad B^{i}=-\frac{1}{4} \Delta^{-2} \varepsilon_{i j k} D T_{j \ell} T_{\mathrm{km}} \mu^{\ell} \mu^{m}$,

$C^{i}=\frac{1}{4} \Delta^{-1} T_{i j} F^{j}$.

Following similar arguments to those we presented in Sec. III, we conclude here that performing the truncation is consistent, provided we have

$T_{i j}=\delta_{i j}, \quad m=-\frac{1}{4} g, \quad B^{i}=0, \quad C^{i}=\frac{1}{4} F^{i}$.

Going back to (6.4), we now find $\alpha^{\prime} \varphi^{\prime}=6 \alpha \varphi$, and hence from (5.6), with the truncation $\psi=0$ that we have made, we find the Pauli metric reduction Ansatz (6.5) reduces to

$$
d \bar{s}_{6}^{2}=e^{-\frac{1}{2} \phi} d s_{4}^{2}+\frac{1}{4} g^{-2} e^{\frac{1}{2} \phi} D \mu^{i} D \mu^{i} .
$$

The field strengths reduce according to the Ansätze

$$
\begin{aligned}
& F_{(2)}=\sqrt{2} \mathcal{F}_{(2)}=-\sqrt{2} \bar{H}_{(2)}=\frac{1}{\sqrt{2}} g^{-1} \omega_{(2)}-\frac{1}{\sqrt{2}} \mu^{i} F^{i}, \\
& \bar{H}_{(3)}=H_{(3)}-\frac{1}{4} g^{-1} \varepsilon_{i j k} F^{i} \mu^{j} \wedge D \mu^{k} .
\end{aligned}
$$

The scalar field $\phi$ is simply taken to be dependent only on the four-dimensional coordinates.

We may now verify that this reduction scheme does indeed give consistent four-dimensional equations of motion, as a consequence of the six-dimensional equations of motion. First, we check the six-dimensional Bianchi identity for $\bar{H}_{(3)}$, which, from (5.4), is

$$
d \bar{H}_{(3)}=-\bar{H}_{(2)} \wedge \mathcal{F}_{(2)}=\frac{1}{2} F_{(2)} \wedge F_{(2)} .
$$

To calculate $d \bar{H}_{(3)}$, we may employ the useful relations

$$
\begin{aligned}
D F^{i} & =0, \quad \frac{1}{2} \varepsilon_{i j k} D \mu^{j} \wedge D \mu^{k}=\mu^{i} \omega_{(2)}, \\
D^{2} \mu^{i} & =g \varepsilon_{i j k} F^{j} \mu^{k} .
\end{aligned}
$$

After some algebra, we find $d \bar{H}_{(3)}$ gives

$$
\begin{aligned}
d \bar{H}_{(3)}= & d H_{(3)}-\frac{1}{2} g^{-1} \mu^{i} F^{i} \wedge \omega_{(2)}-\frac{1}{4} F^{i} \wedge F^{i} \\
& +\frac{1}{4} \mu^{i} \mu^{j} F^{i} \wedge F^{j},
\end{aligned}
$$

while

$F_{(2)} \wedge F_{(2)}=-g^{-1} \mu^{i} F^{i} \wedge \omega_{(2)}+\frac{1}{2} \mu^{i} \mu^{j} F^{i} \wedge F^{j}$.

Therefore, all the coordinate dependence on the internal 2sphere coordinates $\mu^{i}$ cancels, and (6.14) leads to the fourdimensional Bianchi identity 


$$
d H_{(3)}=\frac{1}{4} F^{i} \wedge F^{i}
$$

To check the other six-dimensional equations of motion, it is useful first to calculate the six-dimensional duals of the fields $\bar{H}_{(3)}$ and $F_{(2)}$. We find

$$
\begin{aligned}
& \bar{*}_{(3)}=\frac{1}{4} g^{-2} e^{\phi} * H_{(3)} \wedge \omega_{(2)}+\frac{1}{4} g^{-1} * F^{i} \wedge D \mu^{i}, \\
& \bar{*}_{(2)}=2 \sqrt{2} g e^{-\frac{3}{2} \phi} * \mathbb{1}-\frac{1}{4 \sqrt{2} g^{2}} e^{\frac{1}{2} \phi} \mu^{i} * F^{i} \wedge \omega_{2},
\end{aligned}
$$

together with $\bar{*} d \phi=\frac{1}{4} g^{-2} * d \phi \wedge \omega_{(2)}$. In these expressions, $*$ denotes the Hodge dual in the four-dimensional metric $d s_{4}^{2}$.

We find that the six-dimensional equation of motion for the scalar field $\phi$,

$$
\begin{aligned}
d \bar{*} & d \phi+\frac{1}{2} e^{\frac{1}{2} \phi} \phi_{*} F_{(2)} \wedge F_{(2)}+e^{\phi} \bar{*} \bar{H}_{(3)} \wedge \bar{H}_{(3)} \\
& -4 g^{2} e^{-\frac{1}{2} \phi} \bar{*} \mathbb{1}=0
\end{aligned}
$$

implies, after nontrivial cancellations of the internal coordinate dependence, the four-dimensional equation

$$
d * d \phi+e^{2 \phi} * H_{(3)} \wedge H_{(3)}+\frac{1}{4} e^{\phi} * F^{i} \wedge F^{i}=0 .
$$

The six-dimensional equation of motion for $\bar{H}_{(3)}$, namely $d\left(e^{\phi} \bar{*}_{(3)}\right)=0$, implies the two four-dimensional equations

$$
\begin{aligned}
d\left(e^{2 \phi} * H_{(3)}\right) & =0, \quad \text { and } \\
D\left(e^{\phi} * F^{i}\right)-e^{2 \phi} * H_{(3)} \wedge F^{i} & =0 .
\end{aligned}
$$

The six-dimensional equation of motion for $F_{(2)}$, namely $d\left(e^{\frac{1}{2}} \phi_{*} F_{(2)}\right)-e^{\phi} \bar{*} \bar{H}_{(3)} \wedge F_{(2)}=0$, reproduces the fourdimensional Yang-Mills equations in (6.22). The sixdimensional Einstein equations should reproduce the four-dimensional equations found above, together with the four-dimensional Einstein equations. They can all be derived from the four-dimensional Lagrangian

$$
\begin{aligned}
\mathcal{L}_{4}= & R * \mathbb{1}-\frac{1}{2} * d \phi \wedge d \phi-\frac{1}{2} e^{2 \phi} * H_{(3)} \wedge H_{(3)} \\
& -\frac{1}{4} e^{\phi} * F^{i} \wedge F^{i} .
\end{aligned}
$$

Note that if we send $A^{i} \rightarrow \sqrt{2} A^{i}, g \rightarrow \sqrt{2} g$, and $\phi \rightarrow-\phi$, the $S^{2}$ Pauli reduction we have constructed here then corresponds to the one in the conventions of [12].

\section{CONCLUSIONS}

In this paper, we have employed a relation between consistent DeWitt (group manifold) reductions and Pauli (coset) reductions that was established in [4], applying it to two instances of $S^{2}$ reductions in supergravity theories. In the first example, we addressed the question of whether there exists a consistent $S^{2}$ Pauli reduction of five-dimensional minimal ungauged supergravity. One might expect, in view of the close parallels between the five-dimensional theory and 11-dimensional supergravity, that there could exist such a consistent reduction, paralleling the known consistent $S^{4}$ reduction of the 11dimensional theory. Our starting point was the observation that the minimal five-dimensional supergravity can be obtained as a consistent truncation of the fivedimensional supergravity that one obtains from an $S^{1}$ Kaluza-Klein reduction of a minimal six-dimensional supergravity. By performing a (necessarily consistent) DeWitt reduction of the six-dimensional theory on the $S^{3}=S U(2)$ group manifold, and then reducing this on the $U(1)$ Hopf fibers of the $S^{3}$, one obtains a (necessarily consistent) Pauli $S^{2}$ reduction of the untruncated fivedimensional theory. This does not yet establish the consistency of the Pauli $S^{2}$ reduction of the minimal five-dimensional supergravity; however, since for this to work, the truncation that is still needed in five dimensions would have to be compatible with the Pauli/DeWitt relation. We showed that in fact one cannot consistently perform the truncation of fields in five dimensions; thus, leading to the conclusion that a consistent Pauli $S^{2}$ reduction of five-dimensional minimal supergravity is not possible.

The second example we studied in this paper was concerned with the known consistent Pauli $S^{2}$ reduction of the six-dimensional Salam-Sezgin supergravity. This reduction was derived by direct means [12], and thus although technically much simpler than other consistent Pauli reductions such as the $S^{4}$ or $S^{7}$ reductions of 11dimensional supergravity, the underlying reason for why it should exist remained rather obscure. Our aim in this paper was to try to gain an understanding of the consistency of the reduction by deriving it from a manifestly consistent DeWitt $S U(2)$ group manifold reduction from seven dimensions. A possible candidate for such an explanation was already in existence, since it had been shown in [13] that the Salam-Sezgin theory could indeed be derived via a consistent $S^{1}$ reduction and truncation of a sevendimensional $S O(2,2)$-gauged supergravity. However, this reduction route was not suitable for our present purposes since the Kaluza-Klein vector in the reduction from seven to six dimensions was in fact set to zero, and so it could not supply the needed "twist" that would promote the subsequent $S^{2}$ reduction into an $S^{3}$ reduction from seven dimensions rather than merely $S^{1} \times S^{2}$. 
We then showed that there exists a completely different way of deriving the Salam-Sezgin theory ${ }^{4}$ as a KaluzaKlein $S^{1}$ reduction from the $S O(2,2)$-gauged sevendimensional supergravity, and in this construction, the Kaluza-Klein vector is nonvanishing. The lift of the $S^{2}$ reduction to seven dimensions indeed now gives rise to an $S^{3}$ reduction, and so the possibility of relating the Pauli and DeWitt reductions using the relations established in [4]

\footnotetext{
${ }^{4}$ Or, at least, its bosonic sector. In our present considerations, we have only been concerned with finding an explanation for the consistency of the Pauli $S^{2}$ reduction of the bosonic sector of the Salam-Sezgin theory. The reduction of the fermionic sector will be addressed in [15].
}

arises. The only remaining question is whether the necessary truncation of fields in six dimensions to obtain the pure Salam-Sezgin theory is compatible with the reduction of the DeWitt reduction on its Hopf fibers. In this case, unlike in the first example, we studied in this paper, we found that the truncation is compatible, and so this provides an understanding of the consistency of the $S^{2}$ Pauli reduction of the Salam-Sezgin theory.

\section{ACKNOWLEDGMENTS}

We are grateful to Hong Lü and Ergin Sezgin for useful discussions. This work was supported in part by DOE Grant No. DE-FG02-13ER42020.
[1] N. Straumann, On Pauli's invention of non-Abelian KaluzaKlein theory in 1953, arXiv:gr-qc/0012054.

[2] L. O'Raifeartaigh and N. Straumann, Early history of gauge theories and Kaluza-Klein theories, with a glance at recent developments, arXiv:hep-ph/9810524.

[3] B. de Wit and H. Nicolai, The consistency of the $S^{7}$ truncation in $D=11$ supergravity, Nucl. Phys. B281, 211 (1987).

[4] M. Cvetič, G. W. Gibbons, H. Lü, and C. N. Pope, Consistent group and coset reductions of the bosonic string, Classical Quantum Gravity 20, 5161 (2003).

[5] H. Nastase, D. Vaman, and P. van Nieuwenhuizen, Consistent nonlinear $\mathrm{KK}$ reduction of 11-d supergravity on $\mathrm{AdS}_{7} \times S^{4}$ and selfduality in odd dimensions, Phys. Lett. B 469, 96 (1999).

[6] H. Nastase, D. Vaman, and P. van Nieuwenhuizen, Consistency of the $\mathrm{AdS}_{7} \times S^{4}$ reduction and the origin of self-duality in odd dimensions, Nucl. Phys. B581, 179 (2000).

[7] M. Cvetič, H. Lü, C. N. Pope, A. Sadrzadeh, and T. A. Tran, $S^{3}$ and $S^{4}$ reductions of type IIA supergravity, Nucl. Phys. B590, 233 (2000).
[8] A. Baguet, O. Hohm, and H. Samtleben, Consistent type IIB reductions to maximal 5D supergravity, Phys. Rev. D 92, 065004 (2015).

[9] A. Baguet, C. N. Pope, and H. Samtleben, Consistent Pauli reduction on group manifolds, Phys. Lett. B 752, 278 (2016).

[10] B. S. DeWitt, in Relativity, Groups, and Topology, Les Houches Lectures 1963 (Gordon and Breach, New York, 1964).

[11] A. Salam and E. Sezgin, Chiral compactification on (Minkowski) $\times S^{2}$ of $N=2$ Einstein-Maxwell supergravity in six-dimensions, Phys. Lett. 147B, 47 (1984).

[12] G. W. Gibbons and C. N. Pope, Consistent $S^{2}$ Pauli reduction of six-dimensional chiral gauged Einstein-Maxwell supergravity, Nucl. Phys. B697, 225 (2004).

[13] M. Cvetič, G. W. Gibbons, and C. N. Pope, A string and M-theory origin for the Salam-Sezgin model, Nucl. Phys. B677, 164 (2004).

[14] H. Lü, C. N. Pope, and E. Sezgin, $S U(2)$ reduction of six-dimensional $(1,0)$ supergravity, Nucl. Phys. B668, 237 (2003).

[15] A. Azizi (to be published). 